curve. They can well involve supersonic velocities in the atmosphere and may be the explanation of the so-called 'supersonic turbulence' reported observationally in super-giants. In my work they have appeared with fairly characteristic periods of about one-eighth of the pulsation period. It would be of interest if such periodicity could be found observationally in supergiants. I would like to add that the techniques which have been successfully applied to calculating the non-linear time dependent motions in Chheids and RR Lyrae stars, can also be applied to a detailed non-linear study of one-dimensional instabilities in convection zones such as the spontaneous generation of radial sound waves.

$F$. Edmonds. The temporal power spectra from lines formed at different depths shown as a slide by Michard shows that the fractional contribution of the oscillatory component of velocity fluctuations is 0.55 at $\tau \simeq 0.5$, and that though this contribution is decreasing with increasing depths, it probably will still be important in the convection zone where $\tau>\mathrm{I}$.

\title{
5. OUTER CONVECTION ZONES IN STARS OF DIFFERENT SPECTRAL TYPES AND LUMINOSITIES
}

\author{
E. Böhm-Vitense \\ (University of Heidelberg)
}

It seems to me that the general situation in the theory of convection zones has not changed very much during the last years. There are two different attitudes towards this problem. Some people try very hard to develop a correct theory of turbulent convection in a medium where the density and the instability changes by orders of magnitude within the unstable layer. Though our understanding of the hydrodynamics has improved quite a bit and we can even draw some nice pictures of convection as we have seen from the very interesting talk of Dr Spiegel this morning, it seems to me that we are still far from having a theory applicable to compute the velocities and the convective energy transport in real stars.

Then there is the other groups of astronomers using the only applicable theory of convection developed so far, namely the so-called mxing length theory with its well-known approximations and uncertainties, trying to find out at least some properties of the convection zones. There have been attempts to improve the mixing length theory (for instance Spiegel ( $x$ )) but its major uncertainty remains, namely the size of the characteristic length.

So what can we say about the outer convection zones under these circumstances?

I would like to concentrate on four points:

I. Origin of instability against convection.

2. Upper boundary of convection zones.

3. Amount of energy transported by convection.

4. Velocities of convection currents to be expected.

I. The well-known criterion for instability against convection is

$$
\nabla_{\mathrm{r}}=\left(\frac{\mathrm{d} \ln T}{\mathrm{~d} \ln P_{\mathrm{g}}}\right)_{\text {radiative equilibrium }}>\left(\frac{\mathrm{d} \ln T}{\mathrm{~d} \ln P_{\mathrm{g}}}\right)_{\text {adiabatic }}=\nabla_{\mathrm{a}},
$$

here $\nabla_{\mathrm{a}}<0.4$ where the sign $<$ applies if an abundant element ionizes. We then have to compute $\nabla_{\mathrm{r}}$. With a temperature-distribution

$$
T^{4}=\mathrm{T}_{e f f}^{4}(\bar{\tau}+q(\bar{\tau}))
$$


and with $\bar{\kappa}=a \cdot P^{b}$, where $b \geqslant 0$ we find

$$
\nabla_{\mathrm{r}}=\frac{1}{4} \frac{(\mathrm{I}+d q / \mathrm{d} \bar{\tau})}{(\bar{\tau}+q(\bar{\tau}))}(b+\mathrm{I}) \bar{\tau}\left\{\begin{array}{l}
T: \text { temperature } \\
P_{\mathrm{g}}: \text { gas pressure } \\
\bar{\tau}: \text { mean optical depth } \\
\bar{\kappa}: \text { mean absorption coefficient }
\end{array}\right.
$$

In deeper layers, where $q(\tilde{\tau})=$ const., we find

$$
\nabla_{\mathrm{r}}=\frac{1}{4} \frac{(b+\mathrm{I}) \cdot \bar{\tau}}{\bar{\tau}+q}, \text { and for } \bar{\tau} \gg q \text { is } \nabla_{\mathbf{r}} \sim \frac{1}{4}(b+\mathrm{I}) .
$$

$\nabla_{\mathrm{r}} \gg 0,4$ for $b \gg \mathrm{I}$, e.g. a steep increase of $\bar{\kappa}$ with $P_{\mathrm{g}}$.

$\bar{\kappa}$ increases steeply in the hydrogen ionization zone.

If $b \sim 0$ we find $\nabla_{\mathrm{r}} \sim 0.25$. Therefore we get also instability for $\nabla_{\mathrm{a}}<0.25$. This may occur in the $\mathrm{H}, \mathrm{HeI}$ and $\mathrm{He} I \mathrm{I}$ ionization zones.

The increase of $\bar{\kappa}$ and the decrease of $\nabla_{\mathrm{a}}$ together give the large instability in the hydrogen convection zones in stars with spectral types $\mathrm{F}$ and later.

Another region of small $\nabla_{\mathrm{a}}$ occurs in the $\mathrm{H}_{2}$ dissociation region for late-type stars.

In early $\mathrm{F}$ and late $\mathrm{A}$ stars another kind of instability may possibly occur in very high layers due to large $\mathrm{d} q / \mathrm{d} \bar{\tau}$ caused by the influence of the strong lines on the radiative equilibrium. This has not yet been studied.

2. With the help of the instability criterion we can compute the optical depths of the upper boundaries of the hydrogen convection zones. They are given in Fig. x. For the Sun the upper boundary occurs at $\bar{\tau} \sim 0,8$, it moves downwards for later spectral types, but upwards for earlier stars and higher luminosities.

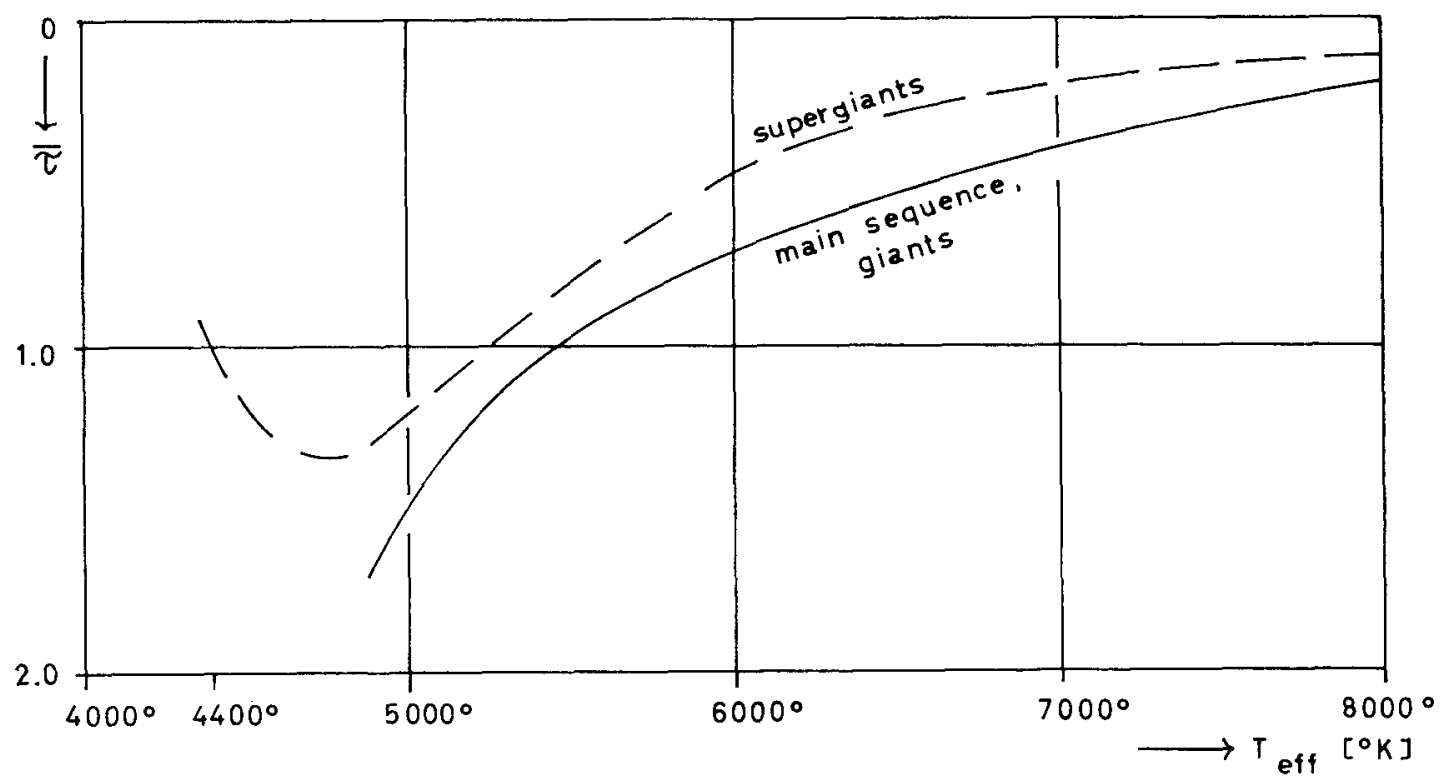

Fig. I. The optical depths $\bar{\tau}$ of the upper boundaries of the hydrogen convection zones as a function of the effective temperature $T_{\text {eff }}$, for main sequence stars and giants (and for super-giants ( $-\longrightarrow$ ). 
3. If we want to estimate the influence of the convection on the temperature stratification we have to know the amount of convective energy transport $\pi F_{\kappa}$. It is given by

$$
\pi F_{\mathbf{x}}=C_{p} \rho \Delta \bar{T} \bar{v}, \quad\left\{\begin{array}{l}
C_{p}: \text { specific heat } \\
\rho: \text { density } \\
v: \text { velocity } \\
\Delta T: \text { temperature fluctuation }
\end{array}\right.
$$

where the mean has to be taken over horizontal planes.

$$
\begin{array}{ll}
\pi F_{\mathrm{K}} \text { will be large if } & \text { a. } \rho \text { is large or } \\
& \text { b. } \Delta T \text { is large or } \\
& \text { c. } v \text { is large. }
\end{array}
$$

$\Delta T$ can be computed from the degree of instability, namely $\nabla_{\mathbf{r}}-\nabla_{\mathrm{a}}$, which determines the difference between the mean temperature gradient of the atmosphere $\nabla$ and the temperature gradient for the moving gas $\nabla^{\prime}$. The schematic Fig. 2 shows the situation. We obtain (2)

$$
\Delta T / T=\left(\nabla-\nabla^{\prime}\right) \cdot l / 2 \mathrm{H},
$$

where $H$ is the scale height, and where $l$ is the mean path length of the gas moving through the layer investigated. $\Delta T$ is large, when $\nabla-\nabla^{\prime}$ is large, e.g. when the radiative exchange is small

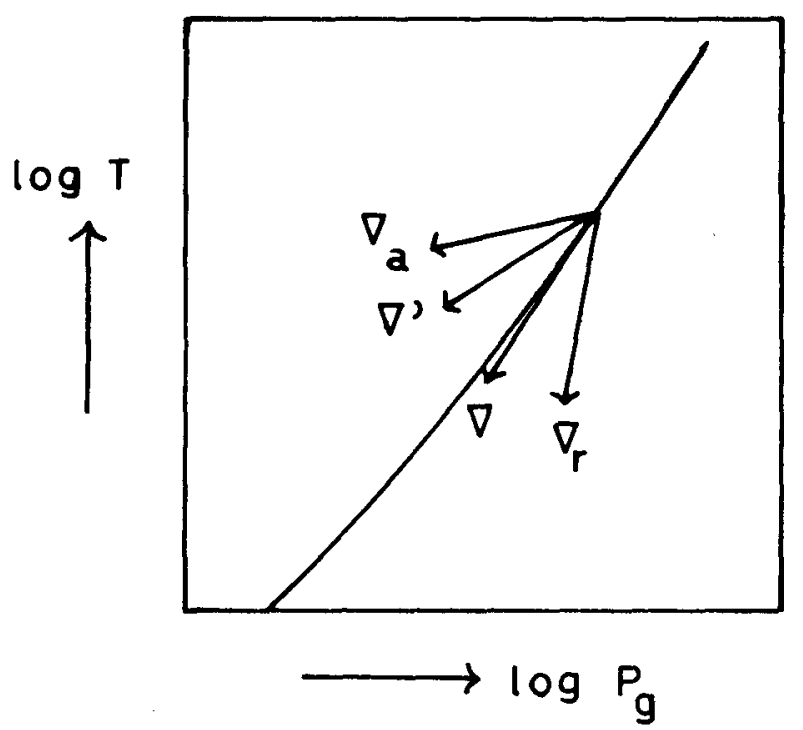

Fig. 2. Schematic diagram of the relative size of the various temperature gradients $\operatorname{d} \ln T / \mathrm{d} \ln P_{\mathrm{g}}$ in the hydrogen convection zone. $\nabla_{\mathrm{r}}=$ radiative gradient, $\nabla_{\mathrm{a}}=$ adiabatic gradient, $\nabla=$ mean gradient actually present, $\nabla^{\prime}=$ gradient of the moving gas considered.

so that $\nabla^{\prime} \sim \nabla_{\mathrm{a}}$, and where the instability is large, e.g. $\nabla_{\mathrm{r}} \gg \nabla_{\mathrm{a}} \cdot v$ is determined by $\Delta T$. We find (2)

$$
\begin{gathered}
\bar{v}^{2}=g \cdot Q \cdot \frac{l^{2}}{4 H}\left(\nabla-\nabla^{\prime}\right) \quad \text { with } Q=\mathrm{I}-\frac{\partial \ln \mu}{\partial \ln T} \\
g=\text { effective gravity, } \quad \mu=\text { molecular weight. }
\end{gathered}
$$

$v$ will also be large for large instabilities and for large pass lengths $l$. 
From these considerations we find that $\pi F_{\mathrm{K}}$ will become small if the radiative energy exchange will prevent a $\Delta T$ to be built up, e.g. in unstable layers close to the surface. It will be very small in the possibly unstable surface layers of $\mathrm{A}$ and $\mathrm{F}$ stars because $\rho$ and $\Delta T$ will be very small. However, in surface layers very small changes in the radiative energy flux $F_{\mathrm{r}}$ may give noticeable values for $\frac{\mathrm{d} F_{\mathrm{r}}}{\mathrm{d} \bar{\tau}}$, which determines the temperature-stratification in these layers.

In deeper layers $\pi F_{\mathrm{K}}$ will generally be larger. It will generally be more important in main sequence stars than in giants and super-giants.

Because of the large values of $\nabla_{\mathrm{r}}-\nabla_{\mathrm{a}}$ in the hydrogen convection zones $\pi F_{\mathrm{k}}$ will be larger there than in the $\mathrm{He} I$ and HeII convection zones. I shall give an example. The radiative energy flux $F_{\mathrm{r}}$ is $\propto \nabla$, and $F_{\mathrm{r}}$ equals the total energy flux $F$ if $\nabla=\nabla_{\mathrm{r}}$. We always have $F=F_{\mathrm{r}}+F_{\mathrm{x}}$ so we find

$$
\frac{F_{\mathrm{K}}}{F}=\mathrm{I}-\frac{\nabla_{1}}{\nabla_{\mathrm{r}}}<\mathrm{I}-\frac{\nabla_{\mathrm{a}}}{\nabla_{\mathrm{r}}} \text { since } \nabla \geqslant \nabla_{\mathrm{a}} .
$$

In the He convection zones we find $\nabla_{\mathrm{r}} \lesssim 0.5$, while $\nabla_{\mathrm{a}} \sim 0.20$, which gives $F_{\mathrm{r}} / F=0.6$. At the bottom of the $\mathrm{He}$ convection zone we may even find $\nabla_{\mathrm{a}} / \nabla_{\mathrm{r}} \sim 0.2 / 0.25$ and therefore $\mathrm{F}_{\mathrm{K}} / \mathrm{F} \sim 0 \cdot 2$.

4. What about the velocities? From the expression for $F_{\mathrm{K}}$ we see that if the total energy is transported by convection, e.g. $F_{\mathrm{K}} \sim F$, then $\bar{v}$ must increase with decreasing $\rho(3)$, and the same is true for $\overline{\Delta T}$. So for stars with active convection we expect to observe larger $v$ for

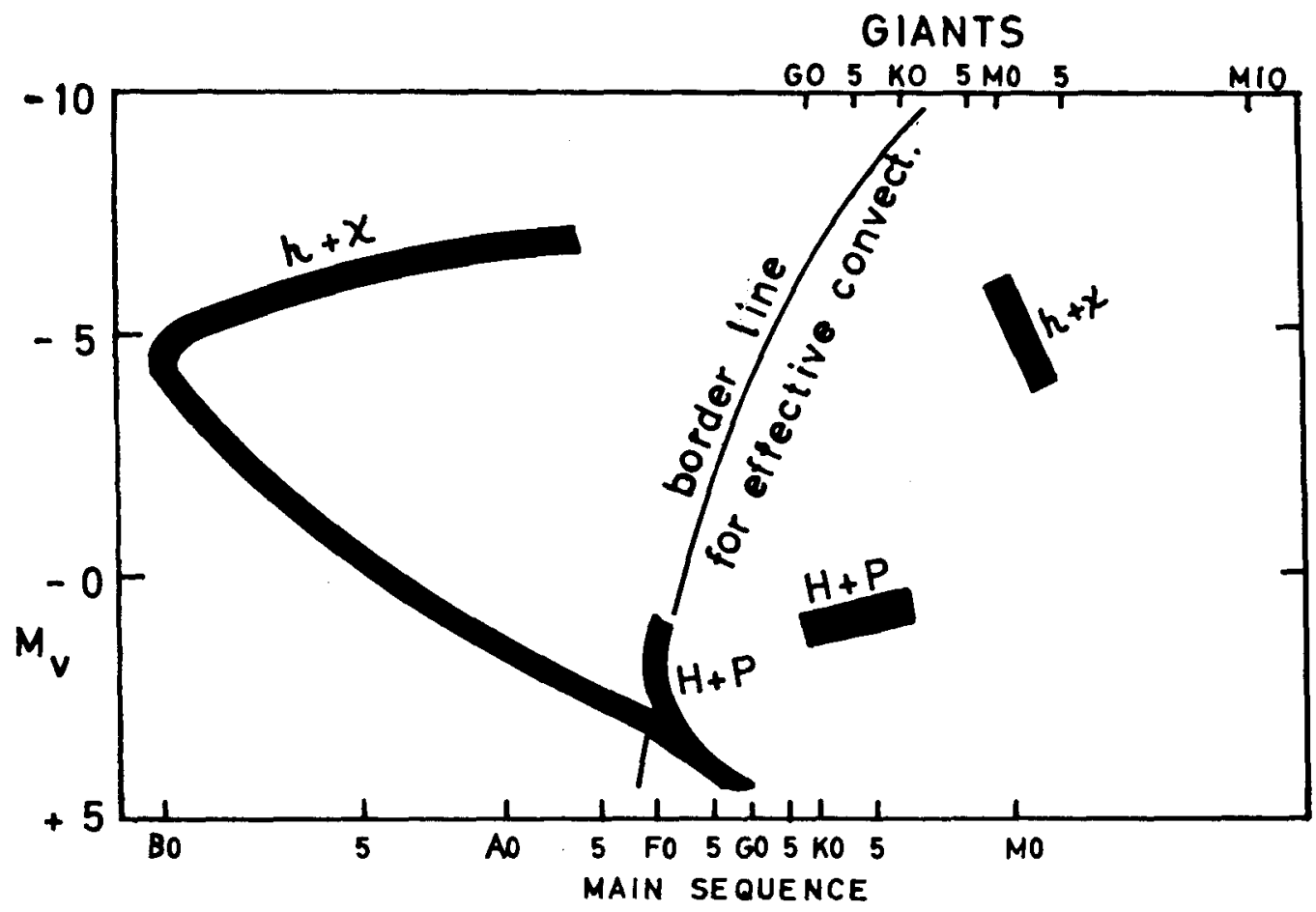

Fig. 3. Colour-magnitude diagram for population I stars (Hyades, Pleiades, $h+\chi$ Persei) with an approximate dividing line for effective convection (-). Above this line no effective convection is expected theoretically. 
giants and super-giants than for main sequence stars and along the main sequence we expect larger velocities for earlier spectral types since the hydrogen ionization occurs in higher layers with smaller $\rho$. At the same time, due to the lower gas pressure, the ionization extends over shallower regions until for A stars the ionization region becomes so shallow that radiative energy exchange and the short path length $l$ prevent an effective convection.

In Fig. 3, we see an H.R. diagram for population I stars, in which I have sketched the dividing line for effective convection. Above this line convection should not be of importance. In fact we can estimate an upper limit for the velocities of the convection currents in $\tau$ Sco to be $\sim 5 \mathrm{~cm} / \mathrm{sec}$. Similar values would be obtained for early super-giants.

The velocities, one would expect to observe are shown in Fig. 4. (Taken from BöhmVitense (4)). They were computed with the help of the mixing length theory.

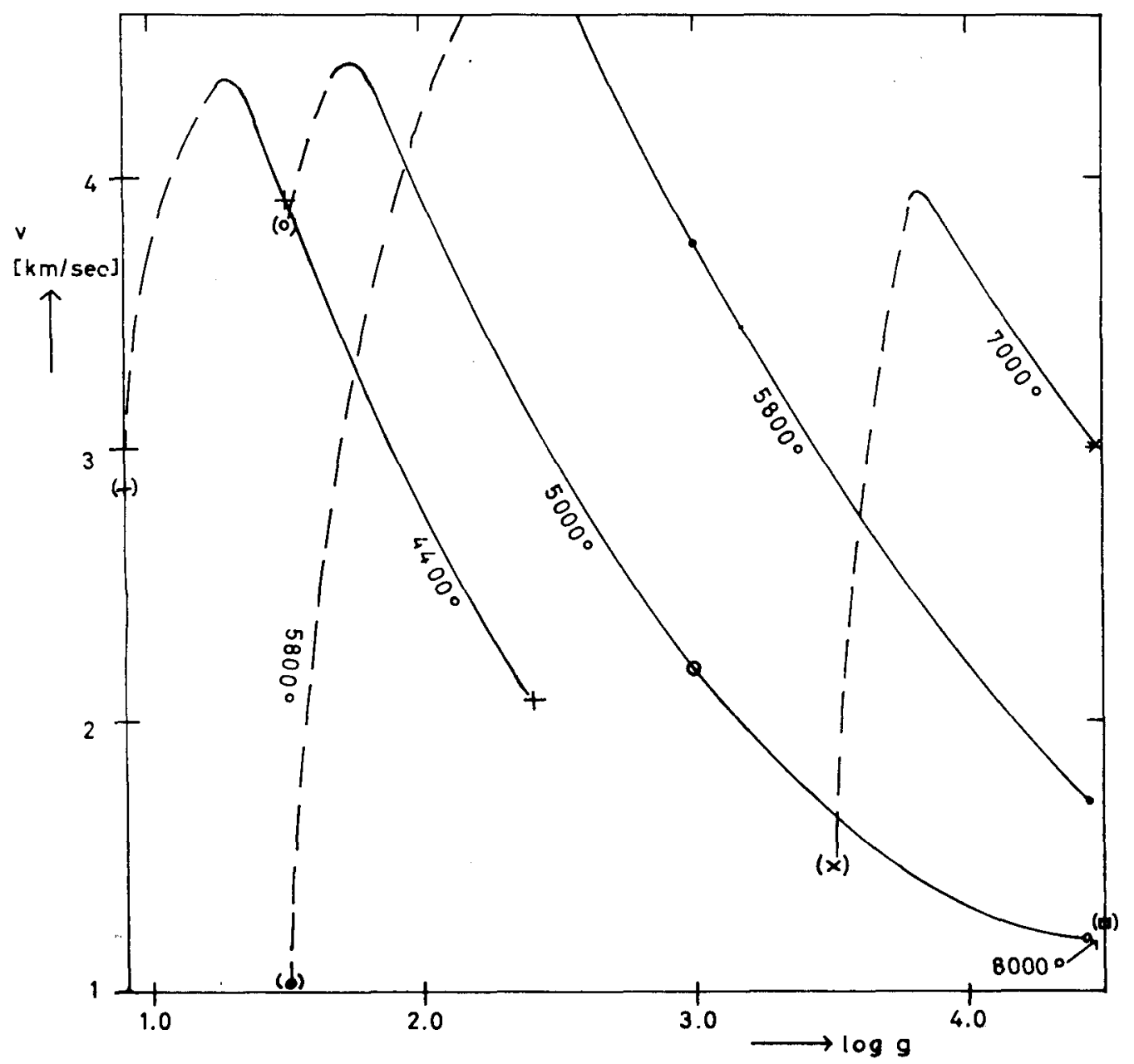

Fig. 4. Mean velocities $v$ of convection currents in the hydrogen convection zones as a function of surface gravity $g$ with $T_{\text {eff }}$ as parameter (computed with the mixing length theory. From Böhm-Vitense (4)). 
With respect to the depth dependence of $v$, we should probably expect a decrease of $v$ from the upper boundary of the convection zone on upwards as was observed by Kegel (5) for $\gamma$ Ser (F6 IV-V) and by Kodaira (6) for HD I6I8I $7\left(T_{\text {eff }}=7630^{\circ} \mathrm{K}\right)$.

No theoretical comparison between population I and population II stars has been made so far. I would guess that larger $\bar{v}$ might be expected for population II stars because of a steeper increase of $\kappa$ with $P_{\mathrm{g}}$.

For population I stars the observations do agree with our expectations for the stars below the dividing line in the H.R. diagram and they are not in disagreement for BV stars (7). But for very early main sequence stars and super-giants we observe line widths which according to Slettebak (8) cannot be explained by rotation and which seem to indicate large turbulent velocities of the order of 20 to $60 \mathrm{~km} / \mathrm{sec}$, increasing with $T_{\text {eff. }}$. These velocities can clearly not be explained by convection currents. Even if one assumes that we observe chromospheric phenomena, we wonder, where the chromosphere should come from, when we do not have an active convection zone.

Kippenhahn (9) has suggested that for rapidly rotating stars turbulent velocities of a few $\mathrm{km} / \mathrm{sec}$ might originate from meridional circulations in the convection zone. But the largest velocities are observed for super-giants which are not the fastest rotators. Perhaps we should first look for the possibilities of other line broadening mechanisms before we look for the origin of supersonic motions in these stars. Could it be that electron scattering might provide such a broadening mechanism?

\section{REFERENCES}

r. Spiegel, E. A. Astrophys. F., 138, 216, 1963.

2. Vitense, E. Z. Astrophys., 32, 135, 1953 .

3. Biermann, L. Astr. Nachr., 264, 361, 1938.

4. Böhm-Vitense, E. Z. Astrophys., 46, 108, 1958.

5. Kegel, W. Z. Astrophys., 55, $22 \mathrm{I}, \mathrm{1} 962$.

6. Kodaira, K. Z. Astrophys., 59, I39, 1964.

7. Wright, K. O. Trans. int. astr. Un., 9, 739, 1955.

8. Slettebak, A. Astrophys. F., 124, 173, 1956.

9. Kippenhahn, R. Z. Astrophys., 48, 172, 1959.

\section{DISCUSSION}

D. Fischel. I have calculated 24 models covering the range of effective temperature equivalent to main sequence stars of Fo to late $G$ with chemical composition ranging from the solar abundance to that of the extreme sub-dwarfs. I have found that the velocity increases with decreasing abundance of the metals in contradistinction to Wallerstein's observations that the velocity parameter from curve of growth determinations decreases with decreasing abundance. So, apparently there is no correlation between the theoretical velocity and the curve of growth velocity parameter.

E. Böhm-Vitense. In interpreting the observed turbulent velocities we should keep in mind that for lines originating in deep layers we shall observe larger scale motions as microturbulence than for lines originating in high layers. I don't know whether this could resolve the difficulties.

C. Cowley. Recently Mrs Cowley and myself made a new curve of growth for the subdwarf HD $\mathrm{I}_{4}$ 0283. We used the new $f$-values of Corliss and Boxman and of Corliss and Warner and the observational data of Aller and Greenstein. The new laboratory data reduced the scatter of the curve of growth considerably so that it was possible for us to say that the turbulent velocity was not zero, as previously supposed, but at the order of $\mathrm{I} \mathrm{km} / \mathrm{sec}$. The same method of treatment gave $\xi_{\mathrm{t}} \odot=\mathrm{I} \cdot 4 \mathrm{~km} / \mathrm{sec}$. We also studied the sub-dwarf HD I9445 
which is somewhat hotter and has a magnetic field of 1000 gauss. The turbulent velocity here was noticeably smaller than in HD I40283, but I would not say that is was definitely zero.

St. Temesvary. I would like to point out that according to an earlier suggestion by Mrs BöhmVitense the velocity of convection as given by the mixing length theory can never reach the velocity of sound. So it seems clear that all observed supersonic velocities are more likely to be ascribed to acoustical phenomena than to the convection directly.

F. Faulkner. I would like to ask whether any attempt has been made to apply the mixing length theory to stars in the low temperature, low luminosity region of the HR diagram. Here I would expect that dissociation of molecular hydrogen would act in a way similar to the ionization of hydrogen in promoting the onset of convection.

E. Böhm-Vitense. Dr Vardya has especially investigated late-type stars. There is indeed another convection zone due to the $\mathrm{H}_{2}$ dissociation.

A. Unsöld. I should like to comment on motions in the early-type super-giant atmospheres. Early-type super-giants (55 Cyg....) show fluctuations in light and radial velocity with characteristic times of some days. The spectra indicate macroturbulence somewhat smaller than the velocity fluctuations and still smaller microturbulence. Bright $H \propto$ points to a kind of extended chromosphere.

The observations can be explained as due to pulsation in many higher modes. The problem is: Why do so early-type super-giants have such noise-like irregular oscillations, while in the somewhat cooler cepheids the fundamental mode is by far the most important.

\section{MICROTURBULENCE ABOVE THE HYDROGEN CONVECTION ZONE \\ F. Roddier}

(Service d'Aéronomie du CNRS, Paris)

We would like to report here a possible interpretation of the profiles of middle strength Fraunhofer lines and especially the profiles of the strontium resonance line we obtained with an atomic beam spectrophotometer. Those profiles are particularly reliable because the experimental technique, which has already been described ( $196 \mathrm{I}$ ), provides a resolution of $10^{7}$, as no instrumental profile correction is required.

Theoretical profiles have been computed with the electronic computer of Meudon. We used models from Meudon and from de Jager (average model I 964). We assumed for a test local thermodynamical equilibrium or LTE. The absorption coefficient has been first computed with a constant microturbulence velocity. In agreement with Allen's result (1949) and Waddell's result ( $195^{8}$ ) we found that a r.m.s. velocity of $x .8 \mathrm{~km} / \mathrm{sec}$ is needed for the profiles at the centre of the disk and more than $3 \mathrm{~km} / \mathrm{sec}$ for limb profiles. But as Schmalberger recently remarked (I963) profiles are rather $V$-shaped at the centre of the disk and rather $U$-shaped near the limb so that computed profiles fit the observations much better near the limb. At the centre of the disk, the agreement with observed profiles is rather poor. As Dr Elste pointed out yesterday, computed wings are too narrow while the computed core is too wide. The difference in the wings is too important to be interpreted as an error on the damping constant.

There are two possible interpretations of the centre to limb increase of the microturbulence velocity. The first one is that turbulence is anisotropic. This was Allen's conclusion in 1949. The second one is a very rapid increase of turbulence with height at a certain level. We shall see that this second possibility leads to much more correct profiles. 